\title{
Larger physical volume with a noncompact lattice regularization of $\mathrm{SU}(\mathrm{N})$ theories
}

\author{
Giuseppe Di Carlo* a ${ }^{*}$ Fabrizio Palumbo ${ }^{\dagger} \mathrm{b}$ and Roberto Scimia ${ }^{\mathrm{c}}$ \\ ${ }^{\mathrm{a}}$ Laboratori Nazionali del Gran Sasso - INFN, I-67010 Assergi (L'Aquila), ITALIA. \\ e-mail address: giuseppe.dicarlo@lngs.infn.it \\ ${ }^{\mathrm{b}}$ INFN - Laboratori Nazionali di Frascati, P. O. Box 13, I-00044 Frascati, ITALIA. \\ e-mail address: palumbof@lnf.infn.it \\ 'Marconi Mobile, Viale dell'Industria, 4-00040 Pomezia (Roma), ITALIA and Dipartimento di Fisica and \\ INFN - Sezione di Perugia, Università degli Studi di Perugia Via A. Pascoli - 06100 Perugia, ITALIA. \\ e-mail address: roberto.scimia@lnf.infn.it
}

Recently it has been found that in a noncompact formulation of the $\mathrm{SU}(2)$ gauge theory on a lattice the physical volume is larger than in the Wilson theory with the same number of sites. In its original formulation such noncompact regularization is directly applicable to $\mathrm{U}(\mathrm{N})$ theories for any $\mathrm{N}$ and to $\mathrm{SU}(\mathrm{N})$ theories for $\mathrm{N}=2$ only. In this work we extend it to $\mathrm{SU}(\mathrm{N})$ for any $\mathrm{N}$ and investigate some of its properties.

\section{Introduction and summary}

One of the major problems in lattice calculations is the need for a large physical volume [1]. One possibility is to adopt an action for which the scaling is established at a larger lattice spacing. In this spirit we investigate a noncompact regularization [2] where exact gauge invariance is enforced by the use of auxiliary fields.

\section{The noncompact regularization for $\mathrm{U}(\mathrm{N})$}

As it is well known, a simple discretization of the continuum covariant derivative breaks gauge invariance. Nevertheless the transformation

$D_{\mu}{ }^{\prime}(x)=g(x) D_{\mu}(x) g^{\dagger}(x+\mu)$

of the lattice covariant derivative $D_{\mu}$ can be exactly enforced by the use of auxiliary compensating fields.

Let us assume the following definitions:

$$
D_{\mu}(x)=\left[\frac{1}{a}-\sigma_{\mu}(x)+i \chi_{\mu}(x)\right] \mathbb{1}
$$

\footnotetext{
${ }^{*}$ On leave from Laboratori Nazionali di Frascati, INFN

†This work has been partially supported by EEC under TMR contract ERB FMRX-CT96-0045
}

$$
\begin{aligned}
& +\left[i A_{\mu}^{a}-\alpha_{\mu}^{a}(x)\right] T_{a}, \\
F_{\mu \nu}(x)= & D_{\mu}(x) D_{\nu}(x+\mu) \\
& -D_{\nu}(x) D_{\mu}(x+\nu), \\
\mathcal{L}_{Y M}(x)= & \frac{1}{4} \beta \operatorname{Tr} F_{\mu \nu}^{\dagger}(x) F_{\mu \nu}(x), .
\end{aligned}
$$

We emphasize that in such a formulation the measure in the partition function is flat.

In the formal continuum limit the field $\sigma_{\mu}$ becomes invariant and decouples together with $\alpha_{\mu}^{a}$, hence these seem to be the auxiliary fields. To control the decoupling of these fields at the quantum level we introduce the invariant potential (without a continuum analog)

$$
\mathcal{L}_{1}=\beta_{1} \sum_{\mu} \operatorname{Tr}\left[D_{\mu}^{\dagger}(x) D_{\mu}(x)-\frac{\mathbb{1}}{a^{2}}\right]^{2} .
$$

We obtain an $\mathrm{U}(\mathrm{N})$ invariant theory. In general we cannot restrict ourselves to the $\mathrm{SU}(\mathrm{N})$ symmetry by changing the covariant derivative, and at the same time the potential $\mathcal{L}_{1}$ does not generate a mass for the $\chi$-field. Indeed the Ward identities [3] show that no $\mathrm{U}(\mathrm{N})$ invariant potential can generate a mass for both abelian fields. We must therefore break explicitly the $\mathrm{U}(\mathrm{N})$ symmetry in order to give all the abelian fields a diver- 
gent mass. The case $\mathrm{N}=2$ is exceptional because by omitting the fields $\chi_{\mu}, \alpha_{\mu}$ we obtain a SU(2) invariant theory with only one abelian auxiliary field. This case has already been exhaustively studied [4].

\section{The noncompact regularization for $\mathrm{SU}(\mathrm{N})$}

To break the $\mathrm{U}(\mathrm{N})$ invariance of the action we add the potential

$\mathcal{L}_{2}=\beta_{2} \frac{1}{a} \sum_{\mu}\left[\operatorname{det} D_{\mu}(x)+\operatorname{det} D_{\mu}^{\dagger}(x)\right]$

which requires [3] the additional term

$\mathcal{L}_{3}=\beta_{3} \frac{1}{a^{2}} \sum_{\mu} \operatorname{Tr}\left[D_{\mu}^{\dagger}(x) D_{\mu}(x)-\frac{\mathbb{1}}{a^{2}}\right]$.

In conclusion the full classical lagrangian is

$\mathcal{L}_{G}=\mathcal{L}_{Y M}+\mathcal{L}_{1}+\mathcal{L}_{2}+\mathcal{L}_{3}$.

By adopting a definition of the covariant derivative where the abelian fields are in a polar representation

$D_{\mu}(x)=\hat{D}_{\mu}(x) \exp i \phi_{\mu}(x)$,

where

$\hat{D}_{\mu}=\rho_{\mu} \mathbb{1}+\left[i\left(A^{\prime}\right)_{\mu}^{a}-\left(\alpha^{\prime}\right)_{\mu}^{a}\right] T_{a}$,

we find that the lagrangian density is stationary for $\bar{\rho}_{\mu}^{(0)}=0$ and

$\bar{\rho}_{\mu}^{( \pm)}=\frac{1}{4 a \beta_{1}}\left[\left|\beta_{2}\right| \pm \sqrt{\beta_{2}^{2}+8 \beta_{1}\left(2 \beta_{1}-\beta_{3}\right)}\right]$.

We have to choose the couplings so as to have one and only one minimum for $\bar{\rho}_{\mu}=1 / a$; this requirement gives [3]

$\left|\beta_{2}\right|=\beta_{3}, 3 \beta_{1}>\beta_{3}$.

In the tree approximation the masses of the auxiliary fields turn out to be

$$
\begin{aligned}
& m_{\rho}^{2}=\frac{6}{a^{2}}\left(4 \beta_{1}-\beta_{3}\right), m_{\phi}^{2}=\frac{18}{a^{2}} \beta_{3}, \\
& m_{\alpha}^{2}=\frac{8}{a^{2}}\left(2 \beta_{1}+\beta_{3}\right) .
\end{aligned}
$$

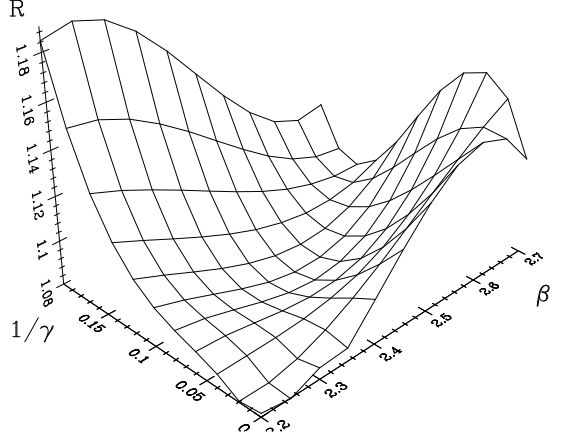

Figure 1. $R(\beta, 1 / \gamma)$ fitting surface.

\section{Numerical study of the SU(2) noncom- pact regularization}

In the $\mathrm{SU}(2)$ case the regularization can be defined with only two coupling constants, namely $\beta, 1 / \gamma$. In the limit of vanishing $1 / \gamma$ we obtain the Wilson regularization.

In perturbative calculations the continuum limit is reached with a scale parameter equal to Wilson one [5].

In numerical simulations the situation is different. On general grounds we expect that there will exist a scaling region in the plane $\beta, 1 / \gamma$ where the properties of convergence to the continuum vary. To investigate this feature we adopted the following strategy [7]. The ratio $R(\beta, 1 / \gamma)$ of two particle masses was calculated on a regular grid in the $(\beta, 1 / \gamma)$ plane. These values were fitted so as to obtain a continuously varying surface. The scaling region is that where the ratio is constant and takes the same value it has in the asymptotic scaling region. Actually in the scaling region the mass ratio must take the same value it has in the asymptotic scaling region for Wilson regularization (the $1 / \gamma=0$ line). The comparison with Wilson results allows us to shorten our calculations, but in no case is mandatory.

It is a crucial condition for the above scheme to be valid, that the only dimensionful quantity of the theory be the renormalization group scaling parameter. This is true if we work in the chi- 


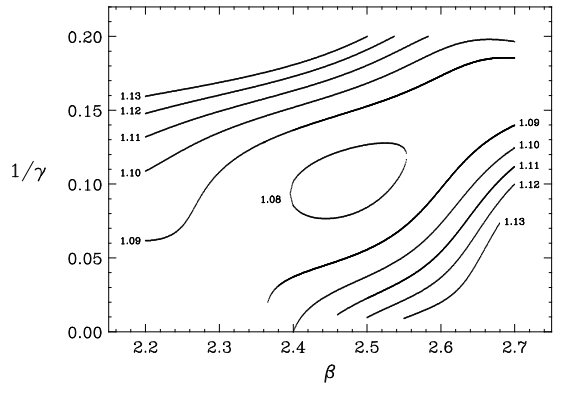

Figure 2. Lines of constant $R$ in the $\beta, 1 / \gamma$ plane.

ral limit, therefore we evaluated the mass spectrum at four finite quark mass values and then we extrapolated it to the chiral limit. The same considerations led us to work in the quenched approximation, also to allow a comparison with the analytical calculations in [5.66.

The details of our numerical work are described in 77 . Here we briefly discuss some of the results. In Fig. 1 we report the fitting surface $R(\beta, 1 / \gamma)$. We see an (almost) flat region (valley) that originates from the $1 / \gamma=0$ line (Wilson results), and propagates towards larger values of $\beta$ for increasing $1 / \gamma$. The flat region in the Wilson limit coincides with the usual asymptotic scaling region for $S U(2)$ pure gauge lattice theory for the small lattices we considered. Therefore we tentatively identify the valley as the scaling window (although not the asymptotic scaling region) for the non-compact regularization. In order to make this observation more precise we report in Fig. 2 the curves of constant $R$ in the plane $\beta, 1 / \gamma$. We performed some additional check to confirm the identification of the scaling region, obtaining reassuring results [7].

Any point inside the scaling window is a good one for approximating the continuum theory, but actual results can be different. In particular the physical value of the lattice spacing, and then the physical volume of the lattice, varies from point to point. We present in Fig. 3 the behaviour of the lattice spacing, as extracted from the $\rho^{+}$mass, along the center of the valley. We can clearly

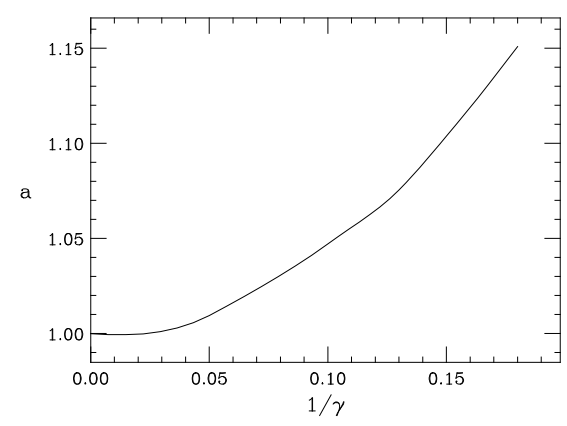

Figure 3. Ratio of non-compact and Wilson lattice spacing along a curve lying on the bottom of the scaling valley.

see that the lattice spacing becomes larger and larger the more we depart from the Wilson case, $1 / \gamma=0$. This improvement can be increased if we move towards larger $\beta$ and $1 / \gamma$, but with a narrower scaling valley.

\section{REFERENCES}

1. R. Petronzio, Nucl. Phys. B (Proc. Suppl.) 83-84 (2000) 136; D.K.Sinclair, Nucl. Phys. B (Proc. Suppl.) 47 (1996) 112; L.Lellouch, Nucl. Phys. B (Proc. Suppl.) 94 (2001) 142.

2. F. Palumbo, Phys. Lett. B244, (1990) 55.

3. F. Palumbo and R. Scimia, hep-lat/0105029

4. C. M. Becchi and F. Palumbo, Phys. Rev. D44 (1991) R946.

5. C. M. Becchi and F. Palumbo, Nucl. Phys. B388 (1992) 595.

6. F. Palumbo, M. I. Polikarpov and A. I. Veselov, Phys. Lett. B297 (1992) 171; B. Diekmann, D. Schutte, and H. Kroger, Phys. Rev. D49 (1994) 3589; B. Borasoy, W. Kramer and D. Schutte, Phys. Rev. D53 (1996) 2599.

7. G. Di Carlo and R. Scimia, Phys. Rev. D63 (2001) 094501 , hep-lat/0009019. 